\title{
Using a Collaborative Approach to Tobacco Control Efforts in Marginalized Communities
}

\author{
Crystal Robertson*, Hadii M. Mamudu, MaryAnn Littleton, Rafie Boghozian, \\ Daniel Owusu, Candice Collins, Liang Wang and Sreenivas P. Veeranki
}

East Tennessee State University, Johnson City, TN, USA

\section{Objective}

To examine community engagement as a means to strengthen tobacco-related policies and programs use in marginalized populations.

\section{Introduction}

Although significant progress has been made in tobacco control in the United States (US) over the past 50 years, more than $15 \%$ of the population currently use tobacco products. ${ }^{1}$ Tobacco use continues to be the leading cause of preventable death, contributing to over 480,000 deaths and about $\$ 300$ billion in economic costs each year. To achieve the Healthy People 2020 (HP2020) objective of $12 \%$ national adult smoking rate by 2020 , it is important to focus our tobacco control efforts on surveillance and addressing disparities in tobacco use prevalence and tobacco-induced diseases across different subpopulations and geographic areas. ${ }^{2}$ Utah reported the lowest prevalence rate $(9.7 \%$ in 2014), while rates as high as $28 \%$ were identified in central Appalachia. Modern epidemiology is limited in its ability to explain patterns of tobacco use and tobacco-related interventions and policies in these highly prevalent, marginalized environments. Therefore, a combination of quantitative and community-based participatory research (CBPR), as proposed in Public Health 3.0, will expand the scope and reach to address all factors of tobacco use, including cross-sector collaboration and multi-level actions. ${ }^{3}$ This study aimed to comprehensively investigate counties in the Northeast Tennessee region where tobacco use prevalence is disproportionately highest, and to identify regional and culturally specific evidence-based practices for tobacco control. Additionally, the study examined how these practices can be scaled up to address similar high tobacco use and disadvantaged populations elsewhere in the US and worldwide.

\section{Methods}

Grounded by the CBPR framework, a mixed-methods approach triangulated multiple sources of data using a three-prong assemblage of Protection, Prevention, and Cessation, to develop tobacco control recommendations and goals as part of a Population Health Improvement Plan for Tennessee. Information gained from health council discussions, focus groups, interviews, and stakeholder meetings were combined with quantitative analyses of secondary data from Tennessee Department of Health, school-based surveys, and qualitative analyses conducted for descriptive and inferential statistics. All discussions and interviews involving 222 individuals from 91 organizations were recorded and organized using NVivo 10 , thematically coded using grounded theory, and analyzed using descriptive statistics. The results utilized aggregated themes generated from the data.

\section{Results}

Tobacco use in the Northeast Tennessee region comprises cigarette smoking and smokeless tobacco, with increasing uptake of electronic cigarettes across all age groups. Among others, culture of tobacco use and cultivation was identified as the most salient factor for tobacco use. Reducing tobacco use requires a foundation built on informatics, community engagement, and a model for sustainable funding to support infrastructure and program interventions. While state and national policies and programs have received less attention in this region, several effective community-based policies and programs to prevent tobacco use were identified, including incentive programs such as Baby and Me, voluntary smoke-free campus policies by businesses and colleges, $100 \%$ screening programs by hospitals, and nicotine-free employee population. Overall, a total of 25 recommendations were identified, with 14 aimed at protection, four at prevention, and seven at cessation. These recommendations culminated into five overarching goals: Protect the population from tobacco and secondhand smoke exposure through policy enforcement and implementation and counter-marketing; Prevent initiation of tobacco use with comprehensive youth-focused programs that increase knowledge and awareness; Expand access to cessation resources and treatment, especially in high risk populations; Foster collaboration and partnership; and Monitor data for evaluation and validity.

\section{Conclusions}

This is one of the few comprehensive attempts to address the social dynamics of tobacco use and identify population and geographic policies and programs in highly prevalent communities. Among the myriad issues identified, the expansion of surveillance data to inform tobacco policy and culturally-tailored tobacco policies and programs are essential to reduce tobacco use in population subgroups. Combining CBPR with actionable data can spur innovations in local efforts, highlight social determinants of health, and contribute to evidence-based policy. While the results of this study primarily provide in-depth descriptions of central Appalachia's tobacco-related risks and their perceptions of and reactions to tobacco prevention intervention, the policies and programs identified through the process may be more readily adopted and scaled-up to address the disparities in tobacco use and tobacco-induced diseases, particularly pertaining to low-income, disadvantaged, and hard-to-reach populations.

\section{Keywords}

Tobacco; Policy change; Surveillance; Community engagement; Disparities

\section{Acknowledgments}

Centers for Disease Control and Prevention

ETSU College of Public Health

Tennessee Department of Health

\section{References}

1. The Health Consequences of Smoking-50 Years of Progress: A Report of the Surgeon General. Atlanta: National Center for Chronic Disease Prevention and Health Promotion, Office on Smoking and Health; 2014.

2. Healthy People 2020. Washington DC: Office of Disease Prevention and Health Promotion; 2014. Tobacco Use. Available from: https://www.healthypeople.gov/2020/topics-objectives/topic/tobaccouse/objectives.

3. DeSalvo KB. et al. Public Health 3.0: Time for an Upgrade. Am J Public Health. 2016 April;106(4):621-622.

\author{
${ }^{*}$ Crystal Robertson \\ E-mail: crystalr501@gmail.com
}

